Research Article

\title{
Microscopic Traffic Characterization of Light Rail Transit Systems at Level Crossings
}

\author{
Robel Desta $\mathbb{D}^{1},{ }^{1}$ Daric Tesfaye $\mathbb{D}^{2},{ }^{2}$ and János Tóth $\mathbb{D D}^{1}$ \\ ${ }^{1}$ Department of Transport Technology and Economics, Faculty of Transportation Engineering and Vehicle Engineering, \\ Budapest University of Technology and Economics, Muegyetem Rkp. 3, 1111, Budapest, Hungary \\ ${ }^{2}$ Department of Civil Engineering, Institute of Technology, Hawassa University, P. O. Box: 05, Hawassa, Ethiopia
}

Correspondence should be addressed to Daric Tesfaye; darictesfaye77@gmail.com

Received 9 February 2021; Revised 10 May 2021; Accepted 18 May 2021; Published 27 May 2021

Academic Editor: Valeria Vignali

Copyright (C) 2021 Robel Desta et al. This is an open access article distributed under the Creative Commons Attribution License, which permits unrestricted use, distribution, and reproduction in any medium, provided the original work is properly cited.

\begin{abstract}
The movement of the light rail vehicles (LRVs) is highly interrupted at level crossings during peak hour times, especially when the intersections are not regulated by a coordinated signal system. Traffic modelling ensures better understanding and interpretation of complex traffic interactions. This study is aimed at modelling light rail transit (LRT) system operational characteristics at level crossings in Addis Ababa City using VISSIM software. The studied scenarios at Sebategna (All Way Stop Controlled, AWSC) and CMC (Roundabout) level crossings are the baseline without LRT scenario, the actual scenario with collected LRV headways, twice arrival frequency scenario, signalized actual LRV arrival, and signalized twice actual LRV arrival. The relative comparisons among the tested scenarios depicted that significant travel time savings can be achieved in some approaches if more green time is offered to nonconflicting phases during a light rail crossing. Overall, the average additional delays at level crossings increase from the base scenario with increasing light rail crossing frequencies, and delay at the level crossing is the second important variable that contributes to the variability of train travel time at peak hours. If it is a must for an intersection to have a rail road to pass through the median, different options should be verified based on the trade-off between the operational cost of the level crossing and the cost incurred if it is grade separated.
\end{abstract}

\section{Introduction}

Access to employment, education, entertainment, health care, and other services are possible because of the integrated urban transport system. Nevertheless, traffic congestion has become a major problem in many large cities, especially in the inner cities, as a result of the rapid growth in private car ownership in recent years, which triggered the expansion of rail transit systems in urban regions. Congestion level in these areas is also increasing due to the rise in population, economy, urbanization, and suburbanization of population, housing, and jobs [1-3].

Light rail transit (LRT) systems have sparked interest as a viable mass transportation solution in cities with increasing traffic congestion. LRT began as a redesign of streetcar service, allowing for faster speeds by separating it from traffic. The versatility of LRT service is distinguished by its ability to operate independently from other traffic below grade, at grade, on an elevated structure, or alongside road vehicles on the pavement. Level crossings are also unique due to their multijurisdictional nature [4-7]. Sener et al. [8] assert that public mass transit systems such as LRT ensure sustainable transport operation and require interventions to improve societal perceptions.

LRT's attractiveness lies in its potential flexibility, design suitability, and lower implementation costs over a heavy rail system's much higher cost. Heavy rail networks are typically grade separated from adjacent vehicle traffic due to power supply risks and organizational priorities, resulting in extremely high initial capital costs. Light rail systems can be integrated into a variety of land use environments, ranging from suburban to high-density central business district (CBD) areas, and can run on a variety of right-of-way styles [9-11]. Policymakers should justify the costs and benefits of 
a crossing safety improvement project to compile information for communicating with the public and government agencies [12].

Integrating LRT systems in existing road corridors has the benefit of plummeting the demand for private vehicles, increasing the safety of train users, reducing pollution of air, waiting time, travel cost, and time of train users. However, it may induce additional delays on vehicles, pedestrians, and other public transport users due to the interval at the crossway and at-grade crossings, and mobility preemption. Priority given to LRV will also cause additional delays and if delays are unacceptable, transport and traffic management strategies are required to improve the traffic situation and pedestrian crossings with an acceptable threshold [13-15]. Policymakers should justify the costs and benefits of a crossing safety improvement project to compile information for communicating with the public and government agencies [12].

The disparity between the minimum travel time and the real travel time, or the difference between the planned and actual travel time, is the basic definition of delay. Delay is sometimes used as a capacity metric; however, delay is a measure of service quality, not capacity, and the relationship between the two is complex. Both scheduled and unscheduled incidents will cause train delays, where scheduled delays are built into the schedule as buffer time, while unscheduled delays are stochastic and a major contributor to network unreliability and volatility. The added delay faced by vehicular traffic as a result of LRVs crossing the highway may be used to assess the potential effect of the LRV on crossing automotive traffic. Delay can be used as a metric to compare the effect to that of other crossings. Different train types/ heterogeneity has different operational characteristics that influence how much time a train is delayed $[11,16]$.

Overall, the addition of LRT crossing alters the flow from uninterrupted to signalized. As traffic volume increases, the capacity of the roadway is reached rapidly and in an uninterrupted flow scenario, total delays increase. As demand exceeds capacity at a signalized intersection, however, total flow remains constant at the saturation flow rate, resulting in a smaller increase in total delay. As traffic flow in a roadway without LRT exceeds its capacity, the average total delay increases, bringing the average total delay closer to that of LRT crossings. As a result, reducing the average additional delay, which is the difference between the two delay values, is critical $[9,17]$. This study is aimed at evaluating the operational characteristics of level crossings using the VISSIM microsimulation model and examining the impact of level crossings on the travel time characteristics of the LRT system.

This paper's composition is as follows: Section 2 discusses studies on the use of traffic modelling techniques, LRT operational analysis, and a review of the literature on the effects of level crossings on various LRT hypothetical scenarios. The methodology and data collection used to create the simulation models for evaluating the impacts of the various developed scenarios are described in Section 3. The outcomes of the different types are presented in Section 4 along with a discussion of the findings. Finally, the conclusions section summarizes the brief discussion of the implications of the results and suggests potential study directions for transit networks.

\section{Literature Review}

Reliable evaluation of existing capacity is indispensable to improve the utilization of existing tracks and to identify areas of bottleneck in the railroad network. Parametric evaluation, simulation, and optimization studies of rail capacity and congestion indicated that statistical approaches could be utilized in place of or in addition to simulation and optimization methods for estimating rail congestion or performance. Railroad productivity has increased over the past quarter century because of technological innovation and improved traffic operations. However, trend analysis using single metrics of capacity such as the commonly used speed and/or reliability metrics fails to capture the complexity of rail performance [18-21].

The most widely used traffic simulation software packages include VISSIM, PARAMICS, Synchro/SimTraffic, and AIMSUN, which are used to accurately reproduce the field observed traffic conditions of LRT volume and signal timing along with systematized outputs for validation of the model [22-24]. Simulation models enable the evaluation of the trade-off between capacity and reliability, which help in obtaining practical capacity measures for effective network operation [25]. For instance, Mitrovic et al. [26] used VISSIM to evaluate traffic operational impacts at LRT atgrade crossings using schedule design with three distinctive scenarios (no-build scenario, one-stop scenario, and onestop-at-busiest-intersection scenario). The results indicated that the third scenario (the best alternative) can save around $9 \%$ in user delay and reduce stops by $10-15 \%$. Studying the factors that affect train delay allows more effective network capacity planning and efficient rail operations. Several researchers also used VISSIM for evaluating multidirectional impacts of autonomous driving on capacity [27], weather effects and emissions along with traffic capacity aspects $[28,29]$, junction operational evaluations [30], and so on.

Chandler \& Hoel [9] found that "on a two-lane isolated intersection, there was an average of 3.6 seconds (hereinafter referred to as "s") of additional delay per vehicle with approximately 250 vehicles crossing and almost 32 seconds/ vehicle (hereinafter referred to as "s/v") of additional delay with 1700 vehicles crossing the light rail tracks. As light rail crossing frequencies decreased (from 5 to 10 minutes), the average additional delay decreased by close to half, to a maximum of $14.9 \mathrm{~s} / \mathrm{v}$. At the 15 -minute crossing frequency, there was a maximum of $9.4 \mathrm{~s} / \mathrm{v}$ of average additional delay, and it was at $7.1 \mathrm{~s} / \mathrm{v}$ with crossings every 20 minutes and a traffic volume of 1700 vehicles/hour (hereinafter referred to as "v/h"). A four-lane isolated intersection case showed that the average additional delays are lower than those for the two-lane case. For lower traffic volumes, between 500 and $3000 \mathrm{v} / \mathrm{h}$ in each direction, the average additional delays range from $2.1 \mathrm{~s} / \mathrm{v}$ to $6.9 \mathrm{~s} / \mathrm{v}$ at the 5 -minute crossing frequency. There is a sharp increase in the total delay to $31 \mathrm{~s} / \mathrm{v}$ at $3250 \mathrm{v} / \mathrm{h}$, and then a slight decrease to around $21 \mathrm{~s} / \mathrm{v}$ as the 
roadway becomes over saturated. When the light rail frequencies decrease, there is a corresponding decrease in additional delay. For instance, at the 20-minute frequency, the maximum additional delay is $8.3 \mathrm{~s} / \mathrm{v}$. The average additional delays for crossing traffic volumes below $3000 \mathrm{v} / \mathrm{h}$ in each direction were all under $2 \mathrm{~s}$ ".

According to [7], the analysis of delay at intersections for three scenarios (considering both light rail vehicle (LRV) and pedestrian crossings, without LRV crossings and LRV crossings in the absence of pedestrians) depicted that the average additional delays due to LRV crossing have been $1.46 \mathrm{~s} / \mathrm{v}$ and $1.51 \mathrm{~s} / \mathrm{v}$ for Sebategna (cross road) and Adey Ababa (cross road) intersections, respectively, while an average additional delay due to pedestrian crossing is shown to be $13.22 \mathrm{~s} / \mathrm{v}$ and $5.37 \mathrm{~s} / \mathrm{v}$ for Sebategna and Adey Ababa intersections, respectively. Based on these results, the traffic performance was more affected by pedestrian crossing than that of LRV crossing.

Disposition of an LRT active priority system for highway-rail grade crossings could significantly reduce the train expected delay with minimal impact on the general traffic. Application of conditional priority control strategy (the timing optimization model for LRT trains) could minimize intersection delays for late trains by $89.5 \%$ and $25.3 \mathrm{~s} /$ train [31-33]. Since delay is considered as the principal metric to select railroad projects, understanding the foremost causes of delay is important to plan capacity more effectively and economically [16].

\section{Materials and Methods}

For developing the simulation models in VISSIM, field data on classified intersection volume by vehicle type, traffic composition, road geometry, categorized average vehicle size, midblock traffic volume, spot speeds, vehicle routing (origin-destination data), LRV arriving frequency, and observations on various parameters of traffic activity were gathered. Moreover, these data were assembled to carry out the required validation.

Furthermore, for statistical samples of vehicles, various data were collected from the field, including actual vehicle classified travel time (the travel time data for the LRT were collected for a statistical number of repetitions), time headway, categorized total traffic delays, and speed data were compiled to ensure that the simulation results are correct as compared to real-world traffic conditions. The collected data were summarized and used to construct the simulation models.

The considered scenarios for experimental modelling, analysis, and processing in each of the simulated conditions are described as follows:

(1) Without LRT: the actual geometric and traffic characteristics are considered without the LRV and the LRT line.

(2) Actual LRV arrival: the actual geometric and traffic characteristics are considered along with the LRV and the LRT line, combining the observed arrival frequency.
(3) Twice actual LRV arrival: the actual geometric and traffic characteristics are considered along with the LRV and the LRT line, incorporating a hypothetical twice arrival frequency.

(4) Signalized actual LRV arrival: the actual geometric and traffic characteristics are considered along with the LRV and the LRT line, combining the observed arrival frequency. Signal program is incorporated for facilitating the movement of the general traffic and LRV.

(5) Signalized twice actual LRV arrival: the actual geometric and traffic characteristics are considered along with the LRV and the LRT line, incorporating a hypothetical twice arrival frequency. Signal program is incorporated for facilitating the movement of the general traffic and LRV.

For the simulation modelling of level crossings, two representative intersections were selected from the total of initially identified ten-level crossings along the LRT line based on their congestion level. Sebategna cross road and LRT line level crossing (Figure 1) was selected from the South-North (SN) line, and CMC roundabout level crossing (Figure 2) was selected from the East-West (EW) line for the simulation. These two intersections were carefully selected from each of the different routes and different intersection hierarchy, since they represent the main features of all intersections in the specific routes and both level crossings have the highest traffic activity.

The selected intersections are comprised of 4-legged unsignalized level crossing and 4-legged roundabout level crossing. The intersections serve as crossroads for major routes that connect the study area's various land use activities. The studied intersections are unsignalized, but traffic flows are regulated by traffic wardens at peak periods. At Sebategna intersection, there are road side hawkers, traders, and retail shops located along the intersecting roads.

There is a huge number of pedestrians, minibuses, and buses at the Sebategna intersection location, which is due to the presence of big bus station close to the intersection; [34] classifies such intersection as All Way Stop Controlled (AWSC). AWSC intersections require drivers on all approaches to stop before proceeding into the intersection. The creation of a right-of-way consensus that alternates between the drivers approaching the intersection occurs, a consensus that is largely based on the intersection geometry and arrival patterns at the stop line.

CMC intersection is located in one of the finest residential and relatively modern establishments with the least population density in the city. Most residents of this location are characterized with better economic status, and hence, automobile ownership and use are significantly high. This high automobile ownership of the location is evinced during the vehicle composition study of the intersection traffic volume. The corridor was selected as it is one of the most congested roads in the city during peak morning and evening travel times. 


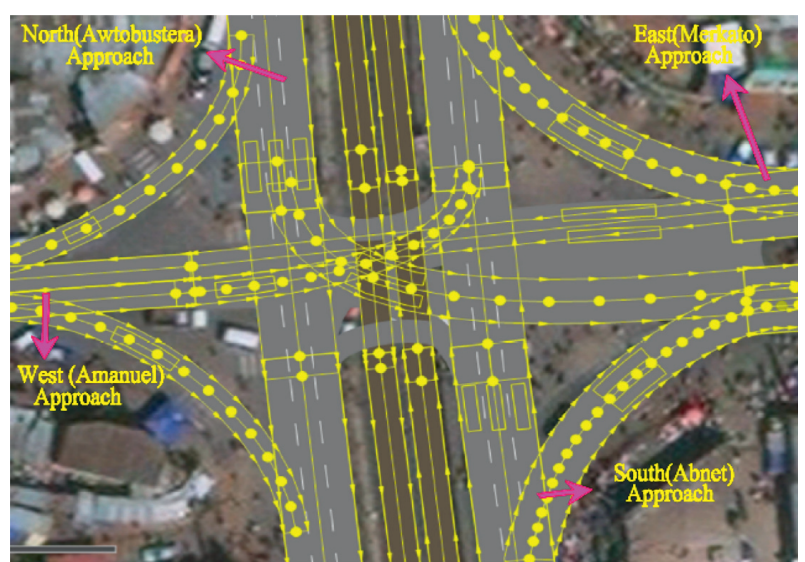

FIGURE 1: Snapshot of the VISSIM model with the links, splined connectors, and reduced speed areas for Sebategna intersection.

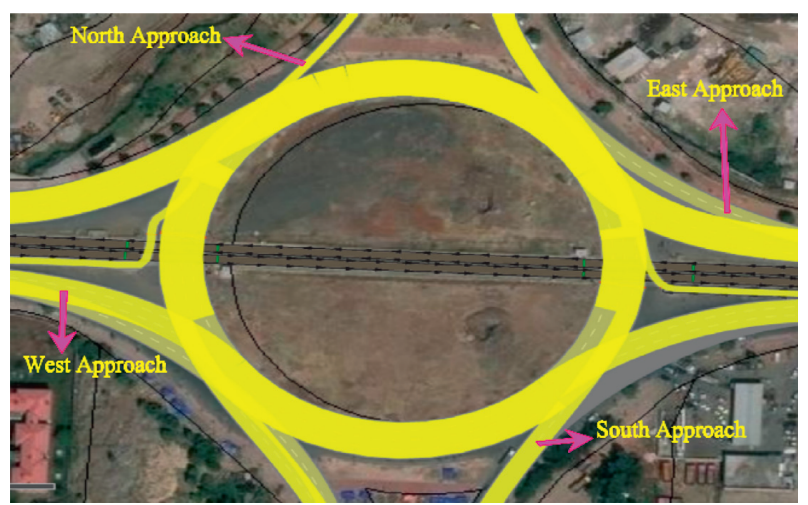

FIGURE 2: Snapshot of the VISSIM model with the vehicle routes for $\mathrm{CMC}$ roundabout intersection.

3.1. Creating Base Models for Simulation Experiments. During the creation of the base model, the field data were recorded in to the work station of VISSIM. The models' links and connectors were drawn on a scaled satellite background area map together with the actual traffic and geometric data (Figure 1).

3.2. Routing Decision and Vehicle Attributes. Vehicle routing (static vehicle route decision) was established in accordance with actual field conditions, taking the flow direction into account (Figure 2). After that, relative flows in each direction were entered for each movement at the intersection. The branches of possible movements, as well as the related traffic volumes, are shown for each routing decision.

New vehicle configurations were specified for those types of vehicles that are not accessible by default in VISSIM to speak to the real vehicle sorts within the city prior to implementing the relative vehicle volumes at each intersection leg.

3.3. Priority Rules and Conflict Areas. "The benefit of the conflict area is that each vehicle determines the total gap time it requires to proceed through the conflict area, based on its own specific vehicle attributes such as acceleration and deceleration capabilities. In addition, with conflict areas, the vehicle that has the right of way is also able to see and respond to vehicles that may have entered the conflict area aggressively and adjust speeds to avoid collisions" [35]. For Sebategna level crossing, the vehicles interaction is managed by conflict areas management tool and the priority for the LRV is given using priority rules.

In comparison to priority rules, conflict areas provide interactive driver behaviour decision making. In this research, the LRV is given full priority at its arrival on the intersection using priority rules. Priority rules are more advised in modelling roundabouts, and those principles are adhered in this study.

3.4. Running Network Simulation. After all the necessary measured inputs are fed for each condition, the networks were saved and ready for simulation. Following to the completion of the analysis and the required results are generated for the given volume of traffic (Figure 3).

Node evaluation, data collection points, and travel time are the three most important evaluation tools used for presentation of the analysis results and validation process. Within a model's calibration area, data collection points must be positioned at all entrance and exit points. For calibration and alternative assessment, archival speed and volume data were used. The data collection points must then be positioned in the model in the same position as they were in the field. Positioning these points should be done with the consideration that adding more points would possibly impact the ability to auto group and any spreadsheets used to handle the outputs.

3.5. Calibration. The calibration step involves determining appropriate parameter values to ensure that the model replicates local traffic conditions as accurately as possible, in order to achieve sufficient model reliability or validity. Then, the model can be used to determine future improvements in the transportation network with trust. Geometry, traffic demand, driver behaviour, signal timing, and other factors could all alter. As calibration method necessitates a detailed examination of real-world data, it is usually limited to current conditions scenarios [9].

3.5.1. Minimum Requirements. As stated in [35], there must be at least two calibration targets based on two separate indicators of effectiveness for the calibration process to be successful. At the very least, all traffic models should use the measure of effectiveness as calibration goals; the most common measures of effectiveness are traffic volumes and speed/travel times.

3.5.2. Throughput Volumes. Primary indicator of calibration is the consistency of throughput volumes from the field matches the simulation output volumes. Simplistic variation in percentage is insufficient to compare the model's wide range of mainline segment or turning movement throughput volumes. The GEH formula is the finest general 


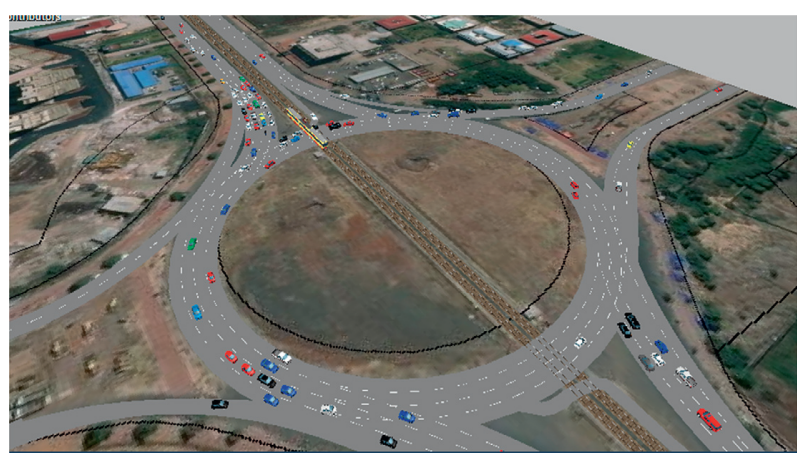

Figure 3: Snapshot of the CMC level crossing during simulation run.

measure for comparing field data and simulation outputs as described in (1). To overcome the broad range of volume data mentioned in the previously, this continuous volume tolerance formula was established. For all principal segments and ramps found in the scope of work, GEH statistics must be determined.

Based on [35], the GEH formula for hourly throughput volumes is

$$
\mathrm{GEH}=\sqrt{\frac{2(m-c)^{2}}{(m+c)}},
$$

where $m=$ output traffic throughput volume from the simulation model $(\mathrm{veh} / \mathrm{h})$ and $c=$ traffic throughput volume based on field data (veh/h).

Calibration of the model uses the GEH formula, which is calculated to an amount of 3 or lesser for high strength and a value above 5 is unacceptable. The above GEH formula was designed to calculate total hourly volumes (vph), using hourly lane volumes, regardless of the facility type, makes it easier to target areas of congestion, and VISSIM also admits input traffic parameters in hourly basis. According to [35], guidance has been formulated on explaining the estimated GEH statistic for the calibration of the throughput traffic volume.

As calibration methodology necessitates the collection of real-world data, it is usually done alone for the current situation scenario. The GEH statistics illustrates that the results for both CMC and Sebategna level crossings are less than three, which are well below the acceptable fit range (Table 1).

3.5.3. Speed. Following the calibration of the throughput volume yields in the model, replication of driver behaviour is required. Matching spot speeds is one process, and mostly this applies to freeway segments, since estimating precise speed data on arterials is hard because of signalized crossings.

When attempting to reproduce speeds in the model, the system used to obtain field measured speed data should be considered (Figure 4). For instance, if spot speeds are gathered by means of a radar speed gun in the field at a specific position, a data collection point should be used to acquire identical model speed data at the matching site. The speed information can likewise be estimated and thought about at different time spans, and this will probably be directed by the accessible field information. HCM [34] states that when contrasted with the noticed genuine spot speed information as an adjustment edge, spot speeds in the model for local roadways with disrupted flow can be permitted to fluctuate by $10 \%$ of the base free flow speed.

The actual average travel time over the study segment is done for three vehicle classes at both intersections. For CMC level crossing $93 \%$ of the vehicle and for Sebategna level crossing $87 \%$ are composed of these vehicles; as a result, the speed represents most part of the vehicle classes. The compared average speed variation of field data and VISSIM output based on the speed validation criteria are under $10 \%$ (Figure 5), which shows the model is acceptable representation of the real traffic behaviour (the calculated percentage changes were all under $8 \%$ ).

3.6. Validation of the Simulation Model. Validation is the method of assessing whether or not the simulation model correctly reflects the system under investigation. Hence, it ensures that the model behaviour correctly and consistently reflects the simulated real-world environment. The developed VISSIM model is validated based on Washington State Department of Transportation VISSIM protocol validation criteria. The simulation models' traffic parameters were carefully examined to ensure that the selected measures of effectiveness matched the simulated outputs [35].

3.6.1. Statistical Number of Simulation Runs. In addition to the validation criteria based on percentage change in errors discussed above, confidence level, as demonstrated in the subsequent section, is envisioned to reveal that the microsimulation runs that have been directed have a normal run, which is representative of the model's actual mean. An initial sampling of the model outputs is needed to assess the degree of confidence in the recorded results. The first sample will be made up of the outcomes of multiple simulation rounds. The quantity of runs must be sufficient to diminish the affect that a normal run will have on the test normal. Individual runs need to use a different set of random number seeds, beginning with one and progressing in order. The stochastic aspects in VISSIM are given a different value of sequence when the random seed is changed, and the traffic flow changes.

The formula in (2) for a 95\% confidence level must be used to ensure that the recorded results are illustrative of the unknown model average [35]:

$$
N=\left[t_{0.025, N-1} \frac{S}{R}\right]^{2}
$$

where $R=$ confidence interval for the true mean, $t_{0.025}$, $N-1$ = Student's " $t$ " statistic for two-sided error of 2.5 percent (totals 5 percent) with $N-1$ degrees of freedom (related to a $95 \%$ confidence level), $S=$ standard deviation about the 
TABLE 1: Sebategna and CMC level crossing volume calibration results.

\begin{tabular}{lcccccccc}
\hline Equation variables & $m$ & $c$ & $m-c$ & $(m-c)^{2}$ & $2 *(m-c)^{2}$ & $m+c$ & $2(m-c)^{2} / m+c$ & $\mathrm{GEH}$ \\
\hline Actual LRT arrival (Sebategna) & 2456 & 2450 & 6 & 36 & 72 & 4906 & 0.01 & 0.12 \\
Actual LRT arrival (CMC) & 5256 & 5475 & -219 & 47961 & 95922 & 10731 & 8.94 & 2.99 \\
\hline
\end{tabular}

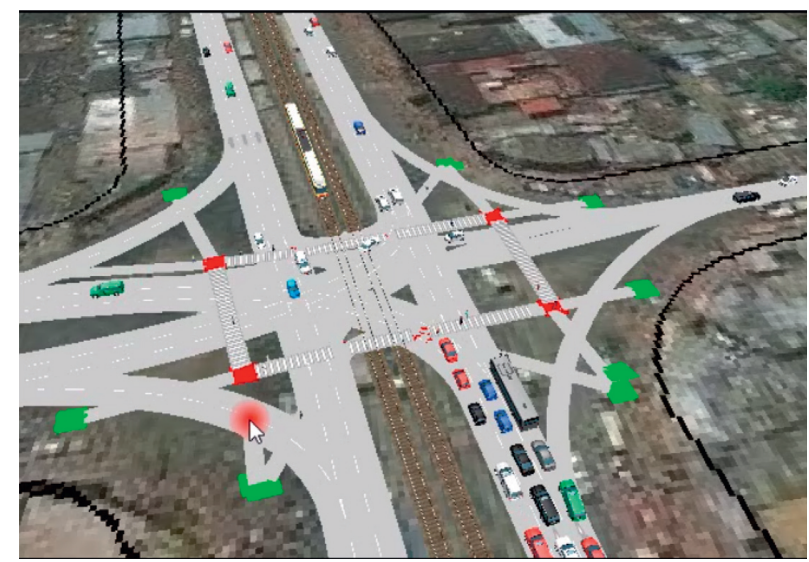

Figure 4: Snapshot display of the Sebategna level crossing during the simulation run for speed data extraction.

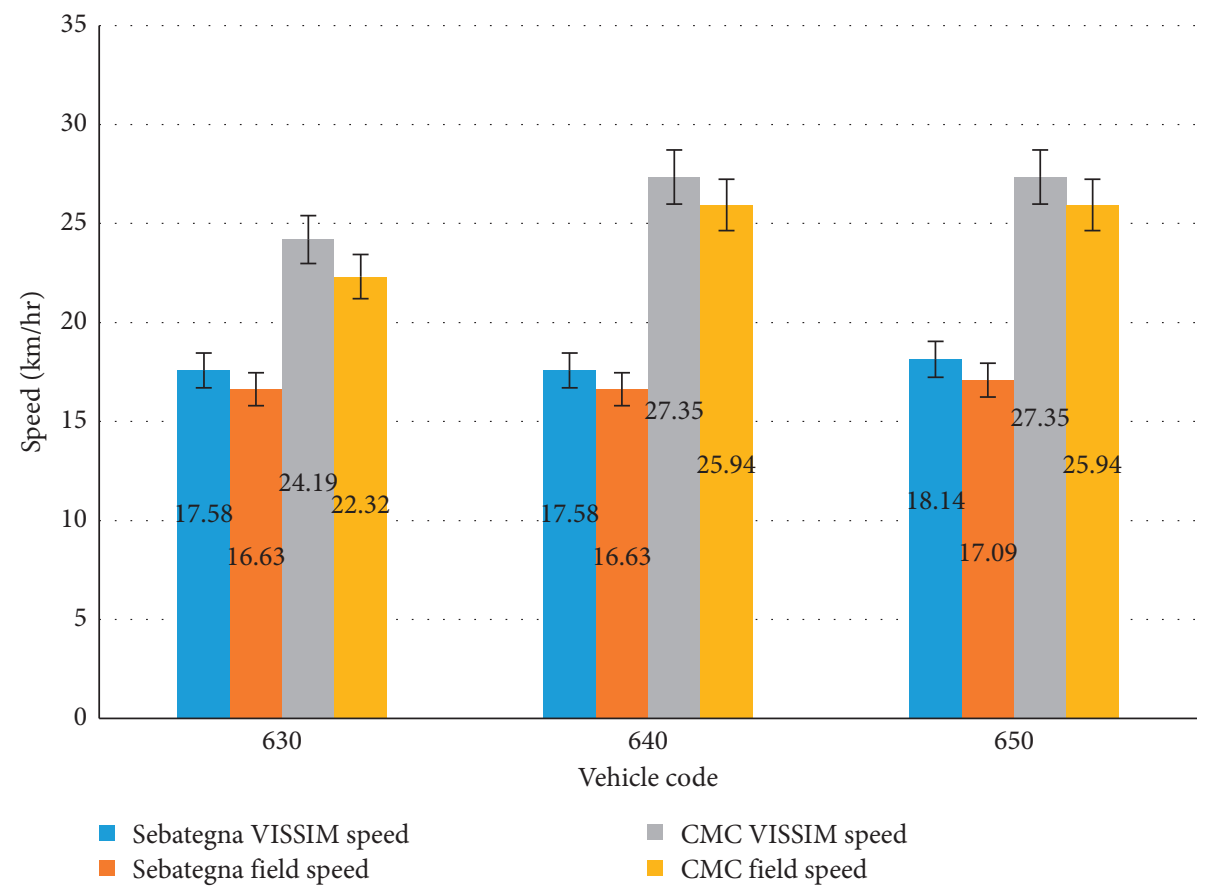

Figure 5: CMC and Sebategna level crossing speed variation.

mean for the selected measure of effectiveness (MOE), and $N=$ number of required simulation runs.

The Student $t$ statistic's value can be found in any statistics book, but $t=2.228$ based on the data set of 11 runs. Unless otherwise stated, a 95\% confidence level must be used.

The aim of this approach is to see if the quantity of runs is adequate to bring an average result that falls within a certain interval of values (confidence interval), where the confidence level is the threshold at which the model's unknown true mean is found. This statistical process requires the generation of an initial data set, which in this case will be the aforementioned 11 runs. In most cases, these 11 runs at $\mathrm{CMC}$ roundabout will generate a large enough sample size to meet our desired confidence criteria (Table 2).

The microscopic experiment runs that have been directed at both junctions have an average volume that is demonstrative of the factual average of the simulation model (Table 2). Provided the inherent differences in results between microsimulation runs (due to the random seed 
TABLE 2: CMC roundabout level crossing average volume of simulation runs.

\begin{tabular}{lccccc}
\hline No. of simulations & Without LRT & Actual LRT arrival & Twice arrival & Signalized actual arrival & Signalized twice arrival \\
\hline 1 & 5610 & 5220 & 5182 & 5616 & 5605 \\
2 & 5621 & 5228 & 5207 & 5627 & 5597 \\
3 & 5631 & 5237 & 5215 & 5632 & 5664 \\
4 & 5638 & 5248 & 5229 & 5610 & 5601 \\
5 & 5622 & 5265 & 5246 & 5665 & 5642 \\
6 & 5617 & 5281 & 5253 & 5676 & 5741 \\
7 & 5602 & 5290 & 5251 & 5683 & 5733 \\
8 & 5611 & 5311 & 5262 & 5695 & 5712 \\
9 & 5622 & 5292 & 5258 & 5693 & 5703 \\
10 & 5722 & 5215 & 5199 & 5716 & 5726 \\
11 & 5731 & 5229 & 5217 & 5724 & 5734 \\
Average & 5639 & 5256 & 5229 & 5667 & 5678 \\
\hline
\end{tabular}

number), each model is obligatory to be evaluated for its reported results to make sure they are indicative of the model and are not biased toward a statistical outlier. Since the true average of the model results is unknown, this is important to ensure that results reported and used in the calibration that follows are representative of the unknown model average.

\section{Results and Discussion}

4.1. Operational Characteristics Based on Delay Results. To obtain a baseline average total delay, each of the intersections in this paper was checked without LRT crossings. The average cumulative delays for each case, which included a number of LRT headways, were then determined. Signal improvements were incorporated to verify if delay improvements can be attained. The difference between the average total delays with two LRT headways, baseline average total delay, and signalized level crossing total delays were compared. The level-of-service (LOS) criteria defined in VISSIM 2020 manual [36] and Highway Capacity Manual [34] are reasonably comparable.

4.2. Sebategna and CMC Level Crossing Results. According to O'Flaherty and Bell [37], the delay that an individual vehicle encounters at a roundabout can be divided into two categories: "moving delay," caused by the vehicle having to slow down, drive a longer distance around a roundabout, and then accelerate back to the road's usual pace and the "obvious delay," caused by the cars having to queue to reach the roundabout, as well as being obstructed by other vehicles when in the roundabout.

4.2.1. Pattern of Traffic Flow and Delay at Sebategna Level Crossing. The average traffic delay in seconds at Sebategna level crossing during the peak period depicted that in the without scenario, the average delay of traffic is $22.31 \mathrm{~s} / \mathrm{v}$ and of which $2 \%, 50 \%, 3 \%$, and $45 \%$ of this delay are experienced by East, West, South, and North approaches, respectively (Table 3). This scenario was used as the base condition for comparison.

In the actual LRV arrival scenario, the average delay was increased by $5.47 \%$ as compared to the base without LRT scenario. The delay absorbed by the East approach had increased, because of the left turn (56.61\%) and the through (16.61\%), which comprise $73.22 \%$ in total are affected by the transit line. The delay absorbed by the West approach increased because $73 \%$ of the vehicles (29\% left turn $44 \%$ through) are affected by the transit line. However, in general, this approach absorbed most of the delay experienced at this intersection and this is mainly because of three basic reasons; this approach has only one lane per each direction, and there is on-street parking of minibus taxis just at the entrance of the approach throughout the peak analysis period and the introduction of the transit line. The delay absorbed by the South approach increased, but not significantly because only 13.55\% (9.14\% U-turn and $4.41 \%$ left turn) vehicles are affected by the introduction of the transit line. The North approach absorbed delay does not increase much because $76 \%$ of the vehicles on this approach are through vehicles and their movement is parallel to the transit movement.

The twice arrival frequency scenario is when the headway of the LRT is reduced by half and the volume of the LRVs on the rail route is twice the actual condition. The average vehicular delay of the intersection is $33.11 \mathrm{~s} / \mathrm{veh}$. The increase in delay is $40.7 \%$ from the actual conditions. From this average vehicular delay, $30 \%$ absorbed by vehicles on East approach, $64 \%$ by West approach, $3 \%$ by South, and 3\% by North approach vehicles. The pattern of traffic flow and delay is almost the same as the actual conditions scenario but there is a slight increase on the East approach and decrease on the rest approaches. This is mainly because $73.22 \%$ of vehicles on the East approach are affected on this scenario, which is relatively higher as compared to the rest of the scenarios.

4.2.2. Impact of Signalizing the Junction at Sebategna Level Crossing. The signal times were regulated for signal synchronization by optimizing the VISSIM simulation results over several attempts (trial and error). The cycle period was $80 \mathrm{~s}$ based on the progressive signal system, and green time for the East was $15 \mathrm{~s}$, while West was given $12 \mathrm{~s}$ green time and $3 \mathrm{~s}$ amber. North and South approaches were given $22 \mathrm{~s}$ green time each and $3 \mathrm{~s}$ amber time. Signal time was given for pedestrians' movement too, with overlapping (nonconflicting) movement of the vehicle with curiosity of checking the effect "what will happen if pedestrians are 
TABle 3: Sebategna level crossing simulation results.

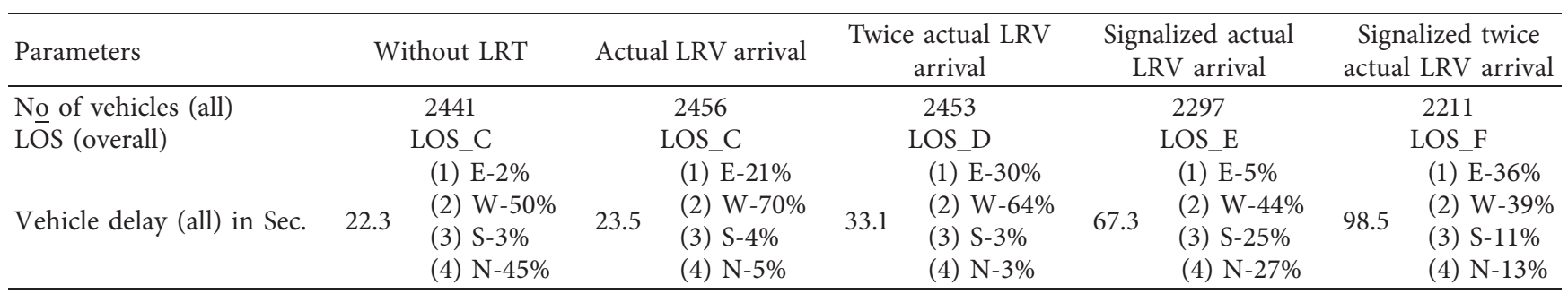

included in the intersection." For the movement of pedestrians on the crossing, the west approach in SN/NS directions (represented by 1) and the movement of pedestrians crossing the East approach in SN/NS directions (represented by 3) were given $50 \mathrm{~s}$ each. The movement of pedestrians crossing the South approach in EW/WE directions (represented by 2) and the movement of pedestrians crossing the North approach in EW/WE directions (represented by 4) were given $30 \mathrm{~s}$ each.

The signal program was applied to the two LRV headway scenarios (actual and twice actual) to evaluate its effect on the average vehicular delay of the level crossings. In the signalized actual arrival frequency scenario, the average delay of traffic was $67.32 \mathrm{~s} / \mathrm{v}$. The installation of traffic signals at the intersection did not improve the delay at the intersection, because the signal increased the stopped time delay of the intersection. Signal with priority to North and South approach redistributed the delay from the East and West approaches to the North and South approaches in the form of stop time delay. Since the vehicle volumes on the South (40\%) and North (35\%) approaches were very high at peak hours, the stop time delay increased the delay of the signalized intersection drastically. Moreover, the high on-street parking, complex geometry, high pedestrian volume, high bus volume, and high volume of U-turn and left turn vehicles of the intersection have created a very complex relationship to create such a delay value.

In the signalized twice arrival frequency scenario, the average delay of traffic was $98.47 \mathrm{~s} / \mathrm{v}$ and the signalized level crossing proposed had increased the average vehicular delay by $197.4 \%$ from the twice arrival unsignalized scenario. The twice arrival of frequency (reduced headway) has increased the delay of the level crossing.

\subsubsection{Pattern of Traffic Flow and Delay at CMC Roundabout} Level Crossing. The analysis reveals that CMC roundabout level crossing recorded higher volume of traffic and delay than Sebategna level crossing (Table 4). In the without scenario, the average delay of traffic was $134.62 \mathrm{~s} / \mathrm{v}$ of which $47 \%$ was experienced by East approach, $5 \%$ by West approach, $32 \%$ by South approach, and $16 \%$ by North approach. This scenario was used as the base condition for comparison.

In the actual scenario, the average delay of traffic was $135.2 \mathrm{~s} / \mathrm{v}$ and the average delay was increased by $0.43 \%$ as compared to the base without LRT scenario. The increase in the average total delay is minimal because on the morning peak traffic volume distribution among the four approaches, i.e., the West approach contributes $18 \%$ of traffic, East approach contributes 39\%, South approach contributes $27 \%$, and the North approach $16 \%$. Because the east approach is parallel to the rail line (not in conflicting direction), the increase in average delay was not that much significant. In this scenario, $53 \%$ of the average delay was absorbed by the vehicles on the East approach, $2 \%$ is absorbed by the vehicles on the West approach, $29 \%$ was absorbed by the vehicles on the South approach, and $16 \%$ was absorbed by the vehicles on the North approach. The South approach is second in the average vehicular delay share, which is due to $76 \%$ of the vehicles arriving in this approach turn left after crossing the rail line; therefore, the movement direction is in direct conflict with the transit line. The North approach is the third in the share of the average vehicular delay. This is because $63.05 \%$ of the vehicles on this approach turn right without entering the roundabout and crossing the rail line. Only $18.10 \%$ percent of the through and $18.85 \%$ of the left turning vehicles of North approach are affected by the transit line. The West approach is the last in absorbing this average vehicular delay, and this is because $54.95 \%$ of the vehicles are through movement, which is parallel to the rail line, and $18.67 \%$ turn right without entering the roundabout with a total of $73.57 \%$ not substantially affected by the transit line.

The twice arrival frequency scenario is when the headway of the LRT is reduced by half and the volume of the LRVs on the rail route is twice the actual condition. The average vehicular delay of the intersection was $136.22 \mathrm{~s} / \mathrm{veh}$. The increase in delay was $0.76 \%$ from the actual conditions. From this average vehicular delay, $47 \%$, $5 \%, 31 \%$, and $18 \%$ were absorbed by vehicles on East, West, South, and North approaches, respectively. The pattern of traffic flow and traffic delay is the same as the actual condition scenario. The increase in average vehicular delay is not significant because of the traffic volume routing that most flows on this intersection with high volume are not in conflict with the transit line. The West, North, and South approaches average vehicular delay has increased, and the delay of East approach has decreased from the actual conditions. This is primarily due to the decrease in headway has affected $26.43 \%$, $36.95 \%$, and $81.3 \%$ of vehicles movements in conflict with the transit line and $77.02 \%$ through movement not affected on the approaches, respectively. 
TABLE 4: CMC level crossing simulation results.

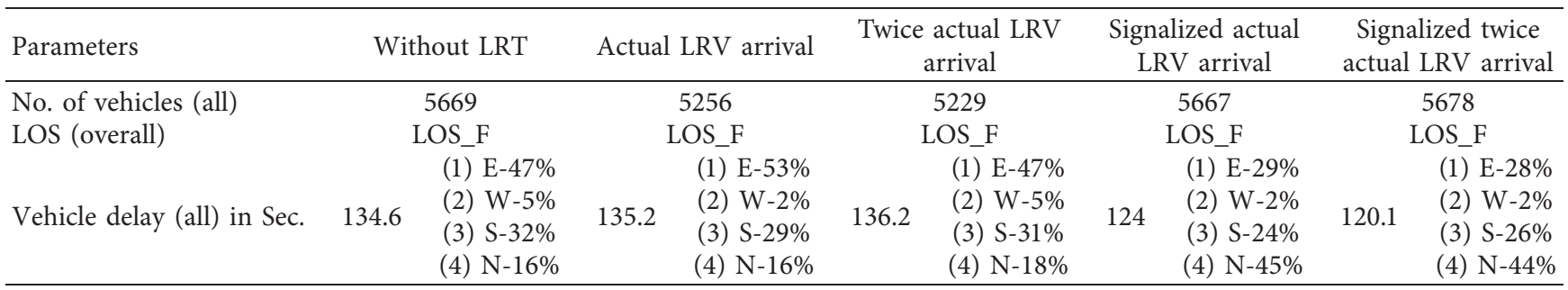

\subsubsection{Impact of Signalizing the Junction at CMC Roundabout} Level Crossing. "Although yield control of entries is the default operation at roundabouts, when necessary, traffic circles and roundabouts can be signalized by metering one or more entries, or signalizing the circulatory roadway at each entry. Roundabouts should never be planned for metering or signalization; however, unexpected demand may dictate the need after installation. Entrance metering can be implemented at the entrance or some distance upstream. Roundabouts operate effectively only when there are sufficient, longer, and acceptable gaps between vehicles in the circulatory lanes. If there is a heavy movement of circulating drivers, then entering drivers at the next downstream entry may not be able to enter. This situation occurs most commonly during the peak periods, and the performance of the roundabout can be greatly improved with entrance metering. A focus on maximizing intersection capacity rather than widening streets may therefore be appropriated" [38].

Many advantages can be achieved with signal control of roundabout, such as through the support of the signals, delay can be well-adjusted in the roundabout and to reduce the delay in coordinated networks via balancing flows and provide the opportunity for transit priority. The queues can be controlled using signals for metering and alleviate the blockage of nearby intersections [39]. The signals are unlit (off) at the roundabout entrance when not active, and this prevents the misperception drivers might came across if they are given a green signal only some distance upstream of the yield control [40].

The signal times were regulated for signal synchronization by optimizing the VISSIM simulation results over several attempts (trial and error). The cycle period was $79 \mathrm{~s}$ based on progressive signal system, and green time for the East approach with the highest traffic volume is given as $40 \mathrm{~s}$ and $13 \mathrm{~s}$ each for the rest of the other approaches. From the $40 \mathrm{~s}$ given for the East approach, $37 \mathrm{~s}$ is green time and $3 \mathrm{~s}$ is amber time. For the rest of the approaches, $10 \mathrm{~s}$ each is given for the green and $3 \mathrm{~s}$ each for the amber.

The signal program is applied to the two LRV headway scenarios (actual and twice actual) to evaluate its effect on the average vehicular delay of the level crossings. In the signalized actual arrival frequency scenario, the delay of the average delay of traffic is $124.01 \mathrm{~s} / \mathrm{veh}$. The signalized level crossing proposed has reduced the average vehicular delay by $8.29 \%$ from the actual unsignalized scenario. This is done by redistributing the existing delays for the less important approaches (less traffic volume). East approach vehicles absorb 29\%, West approach vehicles absorb 2\%, South approach vehicles absorb $24 \%$, and North approach vehicles absorb $45 \%$ of the delay. With this arrangement, the North approach which is the least contributor to the peak hour traffic volume is given most of the delay taken from the East approach which has the highest traffic volume contribution for the peak hour. In this scenario, $29 \%$ of average vehicular delay is experienced by East approach, 2\% average vehicular delay by the West approach, $24 \%$ average vehicular delay by the South approach, and $45 \%$ average vehicular delay by the North approach.

In the signalized twice arrival frequency scenario, the average delay of traffic is $120.13 \mathrm{~s} / \mathrm{v}$. The proposed signalized level crossing has reduced the average vehicular delay by $11.81 \%$ from the twice arrival unsignalized scenario. The pattern of average vehicular delay distribution among the four approaches is not that much different from the preceding scenario, but there is slight increase to the South approach (26\%) and decease of the East (28\%) and North (44\%) approaches. In all the scenarios, when a specific direction is affected, it does not mean that the other direction movements are not affected, because vehicles will be queuing behind the affected direction traffic and the effect is transferred; what it meant to show is the movements in direct conflict with transit line.

4.3. Comparison with Other Studies. Chandler \& Hoel [9] experimented various crossing frequencies on four junction geometries: isolated perpendicular crossings of two- and four-lane roads, an intersection with the light rail line in the median of the main street, and a larger network that includes four at-grade crossings. In spite of the variety of the hypothetical scenarios considered in this paper, comparable results were obtained confirming that light rail vehicle arriving frequency (headway), number of vehicles at the crossing, and the geometry of the intersection have a direct impact on the average rise in wait times experienced by the vehicles. However, unlike the insights provided in Mengesha et al. [7] on AA LRT operation of the existing condition, the developed scenarios in this study provide useful understandings regarding the application and impacts of various viable interventions as potential deployment scenarios. Mitrovic et al. [26] somehow underestimated the impact of the "no-build" scenario, which could be a viable option especially depending on the type of the junction as it is presented for the Sebategna intersection in this study. 
Moreover, future developments as predicted in Wiseman [41] dictate that in the Era of Autonomous Vehicles, rail systems could be obsolete, which again is a controversial topic demanding further investigation.

\section{Conclusions}

This study examined the impacts of level crossings on the variations in the average cumulative delay faced by vehicles. It presented an approach to estimate and compare the additional average delays generated by level crossings with different scenarios (pre- and postdeployment conditions) using VISSIM microsimulation software. Despite the variety of the hypothetical scenarios evaluated in this study, some comparable results were obtained with other studies confirming that light rail vehicle arriving frequency (headway), number of vehicles at the crossing, and the geometry of the intersection have a direct impact on the average rise in waiting times experienced by the vehicles. This study depicted that converting the roundabout level crossing at CMC into signalized junction reduced the average delay by more than $10 \%$. However, signalizing the cross road junction at Sebategna did not improve the delay at the intersection, because the signal program increased the stopped time delay of the intersection, while the twice arrival scenario could give more user satisfaction with minimal impact on the LOS.

Variations in the delay may also rely on the degree of signal synchronization and preemption possibilities when dealing with an intricate highway and crossing function, such as when the light rail is located in the median. The relative comparisons among the tested scenarios depicted that significant travel time savings can be achieved if more green time is specified to nonconflicting phases during the LRT crossing on some of the approaches. Roadways should be operated strategically among the different modes of transport, namely, trains, vehicles, and walking; this synchronization will help in reducing travel time, congestion, and accidents. Policies need to be drafted in such a way that preferred mode of the transport system is prioritized. Therefore, such measures enhance the observed poor operational condition/LOS by considering the balance between user satisfaction, travel time/delay reduction, and the crossing traffic operational performance.

As introducing transit service of any form (rail/bus) into the existing traffic infrastructure is going to be the future of public transportation, the analysis methods need to provide accurate impact on basic measures of effectiveness. If it is must for an intersection to have a rail road to pass through the median, different options and scenarios should be checked based on the trade-off between the operational cost of the level crossing and the cost incurred if it is grade separated. Coordination of highway traffic signal operations with level crossing active warning devices ("railroad preemption") when signalized intersections are located near level crossings is an important measure that will greatly increase the efficiency of a level crossing.

\section{Data Availability}

The data, models, or code generated or used during the study are available from the corresponding author upon request. These data include traffic signal cycle length, traffic composition, road geometry, categorized average vehicle size, midblock traffic volume, actual vehicle categorized traffic data, time headway, categorized total traffic delay, queue length, and various types of traffic simulation outputs from PTV VISSIM.

\section{Conflicts of Interest}

The authors declare that they have no conflicts of interest.

\section{Acknowledgments}

The authors wish to acknowledge all professionals who contributed to this work; especially, Transport Programs Management Office's (TPMO's) Traffic Safety and Management (TSM) division staffs and BRT unit staffs are thanked for their technical assistance and provision of the license for PTV VISSIM 2020. The authors extend their sincere appreciation for the Ethiopian Roads Authority (ERA) and Hawassa University for funding this research work.

\section{References}

[1] ECMT, Managing Urban Traffic Congestion, Organization for Economic Co-operation and Development (OECD) and European Conference of Ministers of Transport (ECMT) Joint Transport Research Centre's Working Group, Paris, France, 2007.

[2] T. Litman, Evaluating Rail Transit Criticism, Victoria Transport Policy Institute, Victoria, BC, Canada, 2017.

[3] A. D. May, "Urban transport and sustainability: the key challenges," International Journal of Sustainable Transportation, vol. 7, no. 3, pp. 170-185, 2013.

[4] C. De Gruyter and G. Currie, "Rail-road crossing impacts: an international synthesis," Transport Reviews, vol. 36, no. 6, pp. 793-815, 2016.

[5] C. Higgins, M. Ferguson, and P. Kanaroglou, "Light rail and land use change: rail transit's role in reshaping and revitalizing cities," Journal of Public Transportation, vol. 17, p. 5, 2014.

[6] H. W. Korve, J. I. Farran, D. M. Mansel, H. S. Levinson, T. Chira-Chavala, and D. R. Ragland, "Integration of light rail transit into city streets. Project A-5 FY'93,” 1996.

[7] D. B. Mengesha, M. Mohammed, and A. Gebissa, "Assessment of the influence of at grade road-rail crossing on traffic performance in addis ababa, case of sebategna and adey ababa road-rail at grade intersection," International Journal of Traffic and Transportation Engineering, vol. 8, 2019.

[8] I. N. Sener, K. Lee, C. P. Durand, A. O. Oluyomi, and H. W. Kohl III, "Intention to use light-rail transit in Houston, Texas, United States: findings from the travel-related activity in neighborhoods study," International Journal of Sustainable Transportation, vol. 14, no. 12, pp. 944-955, 2020.

[9] C. Chandler and L. A. Hoel, Effects of Light Rail Transit on Traffic Congestion, Technical Report Center for Transportation Studies, University of Virginia Charlottesville, Charlottesville, VA, USA, 2004. 
[10] A. L. Habtamu, P. Zhao, and J. Ren, "Track selection for light rail transit (LRT) projects by applying analytic hierarchy process (ahp) decision making method|case study: evaluation of Addis Ababa light rail transit (AALRT) project's track selection," in Proceedings of the Fourth International Conference on Transportation Engineering ICTE 2013: Safety, Speediness, Intelligence, Low-Carbon, Innovation, pp. 634649, Chengdu, China, October 2013.

[11] B. Rymer, T. Urbanik, and J. Cline, "Delay at light rail transit grade crossings," Light, vol. 7, pp. 621-634, 1988.

[12] A. Z. Rezvani, M. Peach, A. Thomas, R. Cruz, and W. Kemmsies, "Benefit-cost methodology for highway-railway grade crossing safety protocols as applied to transportation infrastructure project prioritization processes," Transportation Research Procedia, vol. 8, pp. 89-102, 2015.

[13] D. Cleghorn, A. Clavelle, J. Boone, M. Masliah, and H. Levinson, "Improving pedestrian and motorist safety along light rail alignments," Tcrp report 137, Transportation Research Board, Washington, DC, USA, 2009.

[14] P. Metaxatos and P. S. Sriraj, "Pedestrian safety at rail grade crossings: focus areas for research and intervention," Urban Rail Transit, vol. 1, no. 4, pp. 238-248, 2015.

[15] L. Nithya, A. Aathira, B. Abhilash, J. T. Joel, J. Joseena, and S. Angel, "Pre-feasibility study on light rail transit connecting Kakkanad and Padma junction," in Green Buildings and Sustainable Engineering, pp. 505-515, Springer, Singapore, 2020.

[16] M. H. Dingler, Y.-C. Lai, and C. P. Barkan, "Effect of traintype heterogeneity on single-track heavy haul railway line capacity," Proceedings of the Institution of Mechanical Engineers, Part F: Journal of Rail and Rapid Transit, vol. 228, no. 8, pp. 845-856, 2014.

[17] B. Ogden, "Los Angeles metropolitan transportation authority grade crossing policy: reducing uncertainty and defining scope and cost for light rail transit/roadway crossings," in Proceedings of the Rail Transit Conference American Public Transportation Association, Washington, DC US, August 2004.

[18] M. F. Gorman, "Statistical estimation of railroad congestion delay," Transportation Research Part E: Logistics and Transportation Review, vol. 45, no. 3, pp. 446-456, 2009.

[19] L.-G. Mattsson, "Railway capacity and train delay relationships," in Critical Infrastructure, pp. 129-150, Springer, Berlin, Germany, 2007.

[20] S. Mitra, D. Tolliver, S. Mitra, K. Bachkar, and P. Kayabas, "Estimation of railroad capacity using parametric methods," Journal of the Transportation Research Forum, vol. 49, 2010.

[21] B. A. Weatherford, H. H. Willis, D. S. Ortiz, L. T. Mariano, J. E. Froemel, and S. A. Daly, The State of US Railroads: A Review of Capacity and Performance Data, Rand Corporation, Santa Monica, CA, USA, 2008.

[22] M. Haque and J. D. Sangster, "Best practices for modelling light rail at intersections," 2018.

[23] S. S. Pulugurtha and A. Desai, "Comparative evaluation of Synchro and VISSIM traffic simulation software to model railroad crossings," Computing in Civil Engineering, vol. 23, pp. 184-191, 2007.

[24] K. Shaaban and I. Kim, "Comparison of SimTraffic and VISSIM microscopic traffic simulation tools in modeling roundabouts," Procedia Computer Science, vol. 52, pp. 43-50, 2015.

[25] M. Abril, F. Barber, L. Ingolotti, M. A. Salido, P. Tormos, and A. Lova, "An assessment of railway capacity," Transportation Research Part E: Logistics and Transportation Review, vol. 44, no. 5, pp. 774-806, 2008.
[26] N. Mitrovic, A. Stevanovic, and D. Jolovic, "Evaluating traffic impacts at LRT at-grade crossings in Salt Lake City using schedule design," in Proceedings of the Transportation Research Board 91st Annual Meeting, Washington D.C., USA, January 2012.

[27] V. Zeidler, H. S. Buck, L. Kautzsch, P. Vortisch, and C. M. Weyland, "Simulation of autonomous vehicles based on Wiedemann's car following model in PTV Vissim (No. 1902482)," in Proceedings of the Transportation Research Board 98th Annual Meeting, Washington, D.C., US, January 2019.

[28] C. Chen, X. Zhao, H. Liu, G. Ren, Y. Zhang, and X. Liu, "Assessing the influence of adverse weather on traffic flow characteristics using a driving simulator and VISSIM," Sustainability, vol. 11, no. 3, p. 830, 2019.

[29] K. Shaaban, H. Abou-Senna, D. Elnashar, and E. Radwan, "Assessing the impact of converting roundabouts to traffic signals on vehicle emissions along an urban arterial corridor in Qatar," Journal of the Air \& Waste Management Association, vol. 69, no. 2, pp. 178-191, 2019.

[30] J. Fabianova, P. Michalik, J. Janekova, and M. Fabian, “Design and evaluation of a new intersection model to minimize congestions using VISSIM software," Open Engineering, vol. 10 , no. 1 , pp. $48-56,2020$.

[31] Highway-Rail Grade Crossing Technical Working Group (HRGCTWG), Guidance on Traffic Control Devices at Highway-Rail Grade Crossings, Federal Highway Administration, Washington, D.C., USA, 2002.

[32] M. Li, G. Wu, Y. Li, F. Bu, and W.-b. Zhang, "Active signal priority for light rail transit at grade crossings," Transportation Research Record: Journal of the Transportation Research Board, vol. 2035, pp. 141-149, 2007.

[33] S. P. Venglar, M. S. Jacobson, S. R. Sunkari, R. J. Engelbrecht, and I. Urbanik, "Guide for traffic signal preemption near railroad grade crossing," 2000.

[34] HCM, Transportation Research Board, National Research Council, Washington, D.C., USA, 2010.

[35] WSDOT, Protocol for VISSIM Simulation: VISSIM validation criteria, Washington State Department of Transportation (WSDOT), Olympia, WA, USA, 2014.

[36] A. G. PTV, VISSIM 2020.00 User Manual, PTV Karlsruhe, Karlsruhe, Germany, 2020.

[37] C. A. O'Flaherty and M. G. H. Bell, Transport Planning and Traffic Engineering, Science Direct, Amsterdam, Netherlands, 4th edition, 2003.

[38] B. W. Robinson, L. Rodegerdts, W. Scarborough et al., Roundabouts: an informational guide. Technical Report United States, Federal Highway Administration, Washington, D.C., USA, 2000.

[39] V. W. Inman and G. W. Davis, Synthesis of Literature Relevant to Round about Signalization to Provide Pedestrian Access, Access Board, Washington, D.C., USA, 2007.

[40] B. Baranowski, "Pedestrian crosswalk signals at roundabouts: where are they applicable?," in Proceedings of the TRB National Roundabout Conference, Vail, CO, USA, December 2005.

[41] Y. Wiseman, "In an era of autonomous vehicles, rails are obsolete," International Journal of Control and Automation, vol. 11, no. 2, pp. 151-160, 2018. 